\title{
THE NONEXISTENCE OF A CERTAIN TYPE OF SIMPLE GROUPS OF ODD ORDER
}

\author{
MICHIO SUZUKI
}

1. We consider a finite group $G$, satisfying the following condition (W):

The centralizer of any element $\neq 1$ of $G$ is abelian.

L. Weisner [8] has studied finite groups with this condition (W), and proved that such groups are either solvable or simple. The problem of determining the possible types of simple groups satisfying the condition $(\mathrm{W})$ has not been attacked until quite recently. A few years ago working independently G. E. Wall and the author proved that a nonabelian simple group of even order satisfying the condition (W) is isomorphic with the linear fractional group $\operatorname{LF}\left(2,2^{\mu}\right)$ over a finite field of characteristic 2 . The proof of this theorem will be given elsewhere (see Brauer-Suzuki-Wall [2]).

The purpose of this note is to show that the order of a nonabelian simple group satisfying $(\mathrm{W})$ must be even. Hence the linear fractional groups $\operatorname{LF}\left(2,2^{\mu}\right)$ are the only simple groups which satisfy the condition (W).

As a corollary to this result, we can show the nonexistence of the Redéi group of odd order (cf. Redéi [6]). Here by a Redéi group we shall mean a nonabelian simple group $G$ such that every proper subgroup of a maximal subgroup of $G$ is abelian. Together with the result by Redéi, we can now conclude that the only Redéi group is the alternating group on 5 letters. A generalization of Redéi's theorem will be considered in the final section.

2. If $A$ is a maximal abelian subgroup of a group satisfying the condition (W) of the preceding section, then $A$ satisfies the following property:

\section{$A$ is the centralizer of every element $\neq 1$ of $A$.}

This section will be devoted to a general study of an abelian subgroup of any finite group satisfying the above property. Brauer and Fowler have considered such an abelian subgroup and obtained most of the results in this section (cf. [1]).

Received by the editors December 24, 1954 and, in revised form, November 2 , 1956. 
Let $N$ be the normalizer of $A$ and

$$
n=[A: e] \text { and } l=[N: A] \text {. }
$$

If a conjugate subgroup $\sigma A \sigma^{-1}$ of $A$ contains an element $\rho \neq 1$ of $A$, then the centralizer $N(\rho)$ of $\rho$ contains both $A$ and $\sigma A \sigma^{-1}$. From the assumption on $A, N(\rho)=A$. Hence $\sigma A \sigma^{-1} \subseteq A$. In particular we conclude the following proposition. Two elements of $A$ are conjugate to each other in $G$ if and only if they are conjugate in $N$. Since each element $\neq 1$ of $A$ has exactly $l$ conjugate elements in $N, l$ is a divisor of $n-1$. Thus

$$
w=(n-1) / l
$$

is an integer and $G$ has exactly $w$ conjugate classes $C_{1}, C_{2}, \cdots, C_{w}$ which contain an element $\neq \leq 1$ of $A$. It is easy to see that $N$ has exactly $w$ irreducible characters $\theta_{1}, \cdots, \theta_{w}$ of degree $l$. They are the induced characters induced by nonprincipal linear characters of $A$ and hence vanish on elements of $N$ not in $A$. Since conjugate classes $C_{i} \cap A$ of $N$ are special (cf. [7, Lemmas 4 and 5]), we may consider the "exceptional" characters $\Theta_{1}, \cdots, \Theta_{w}$ of $G$ associated with $\theta_{1}, \cdots, \theta_{w}$ in case $w \geqq 2$ [7, Lemma 5$]$. In fact these exceptional characters are defined by the following property:

If we consider the induced characters $\theta_{i}^{*}$ of $G$, the decomposition of $\theta_{i}^{*}$ into irreducible components takes the form

$$
\theta_{i}^{*}=\epsilon \Theta_{i}+\Delta
$$

where $\epsilon= \pm 1, \Delta$ is a (generally reducible) character of $G$ (or $\Delta=0$ identically) and $\epsilon, \Delta$ are independent of $i$.

We call these characters $\Theta_{1}, \cdots, \Theta_{w}$ the exceptional characters associated with $A$, or simply $A$-characters. The exceptional characters are defined only when $w \geqq 2$. Hence we assume $w \geqq 2$ in the rest of this section.

Exceptional characters satisfy the following properties. Let $D$ be the set of elements in $G$ not conjugate to any element $\neq 1$ of $A$.

(I) $\Theta_{i}(\sigma)=\Theta_{j}(\sigma)$ on $\sigma \in D$ for any pair $(i, j)$. In particular $A$ characters have the same degree.

This may be proved by using the fact that $\theta_{i}^{*}(\sigma)=\theta_{j}^{*}(\sigma)$ on $\sigma \in D$.

(II) The exceptional characters are linearly independent on $C_{1}, \cdots, C_{w}$.

If $\sum_{i=1}^{w} a_{i} \Theta_{i}(\sigma)=0$ for $\sigma \in C_{j}(j=1,2, \cdots, w)$, then using (I) we conclude

$$
\left(\sum_{i=1}^{w} a_{i} \Theta_{i}\right)\left(\Theta_{k}-\Theta_{l}\right)=0
$$


on $G$ for any pair $k, l(1 \leqq k, l \leqq w)$. The orthogonality relations for group characters yield now $a_{k}=a_{l}$. Hence $\sum_{i=1}^{w} \Theta_{i}(\sigma)=0$ for $\sigma \in C_{j}$. On the other hand we have

$$
\sum_{i=1}^{w} \Theta_{i}(\sigma)=\epsilon \sum_{i=1}^{w} \theta_{i}^{*}(\sigma)-\epsilon w \Delta(\sigma) .
$$

Hence $\sum_{i=1}^{w} \Theta_{i}(\sigma)=0$ implies $w \Delta(\sigma)+1=0$ which is impossible $(\Delta(\sigma)$ is an algebraic integer and $w \geqq 2)$.

(III) If $B$ is another abelian subgroup of $G$ satisfying the same condition as $A$, namely satisfying the condition that $B$ is the centralizer of any element $\neq 1$ of $B$, and if $B$ is not conjugate to $A$ in $G$, then $\Theta_{1}, \cdots, \Theta_{w}$ are nonexceptional for $B$.

This is an easy consequence of (I) and (II). If $\Theta_{i}$ is a $B$-character, every $\Theta_{k}(k=1,2, \cdots, w)$ is also a $B$-character, since $\Theta_{i}=\Theta_{k}$ on $B$ by (I). This gives a contradiction to (II) ((II) applied to $B$ ).

Let $\theta_{0}$ be the character of the regular representation of $N / A$. Then $\theta_{0}$ is the induced character of $N$ induced by the principal character of $A$. If $\theta_{0}^{*}$ is the induced character of $G$ induced by $\theta_{0}$, then $\theta_{0}^{*}(\sigma)$ $=\theta_{0}(\sigma)$ for $\sigma \neq 1$ of $A$. Hence

$$
\begin{aligned}
& \sum_{\sigma \in G} \theta_{0}^{*}(\sigma)\left(\Theta_{\imath}\left(\sigma^{-1}\right)-\Theta_{j}\left(\sigma^{-1}\right)\right) \\
&=\epsilon[G: N] \sum_{\sigma \in A} \theta_{0}(\sigma)\left(\theta_{i}\left(\sigma^{-1}\right)-\theta_{j}\left(\sigma^{-1}\right)\right)=0 .
\end{aligned}
$$

This implies the following proposition.

(IV) $\theta_{0}^{*}$ contains $\Theta_{1}, \cdots, \Theta_{w}$ with the same multiplicity.

$\theta_{0}^{*}$ and $\theta_{i}^{*}$ take the same value on $\sigma \in D$. Hence using (IV) we see that

$$
\theta_{0}^{*}-\theta_{i}^{*}=1-\epsilon \Theta_{i}+a \sum_{k=1}^{w} \Theta_{k}+\sum_{\mu} x_{\mu} X_{\mu}
$$

is a linear relation of irreducible characters, which vanishes on every $\sigma \in D$. Here 1 is the principal character and the $X$ 's are the nonprincipal, nonexceptional characters of $G$. It follows from the definition of the induced characters $\theta_{0}^{*}$ and $\theta_{i}^{*}$ that

$$
(1 / g) \sum_{\sigma \in G}\left|\theta_{0}^{*}(\sigma)-\theta_{i}^{*}(\sigma)\right|^{2}=1+l .
$$

Hence using the orthogonality relations we conclude that

$$
l=a^{2}(w-1)+(a-\epsilon)^{2}+\sum x_{\mu}^{2} .
$$


This relation is essential in the proof of our main result.

The coefficients $a$ and $x_{\mu}$ in the relation $\left({ }^{*}\right)$ can be obtained in a second manner. Consider a linear character $\xi$ of $A$. Then the character $\xi^{*}$ of $G$ induced by $\xi$ coincides with one of $\theta_{i}^{*}(i=0,1, \cdots, w)$. Suppose $X$ is a nonexceptional character of $G$, then $X$ is contained in all $\theta_{i}^{*}(i>0)$ with the same multiplicity, say $\alpha$. If the multiplicity of $X$ in $\theta_{0}^{*}$ is $\beta, \theta_{0}^{*}-\theta_{i}^{*}$ contains $X$ with the multiplicity $\beta-\alpha$. Hence $x_{\mu}=\beta-\alpha$ if $X=X_{\mu}$. By the reciprocity law of Frobenius (cf. $[3, \S 246]$ ) the restriction $X / A$ of $X$ to $A$ satisfies the equation

$$
X / A=\beta \xi_{0}+\alpha \sum \xi
$$

on $A$, where $\xi_{0}$ is the principal character of $A$ and the summation of the second term ranges over all nonprincipal irreducible characters. Since $A$ is abelian, every irreducible character of $A$ is linear. Hence comparing degrees in both sides we get

$$
D g(X)=\beta+\alpha(n-1) \equiv \beta-\alpha(\bmod n) .
$$

The orthogonality relation yields now $\xi_{0}+\sum \xi=0$ for any element $\sigma \neq 1$ of $A$. Hence

$$
X(\sigma)=\beta-\alpha \text { on } \sigma \neq 1 \in A .
$$

Thus we get the following proposition.

(V) If $X$ is a nonexceptional character, then $X$ takes a rational integral value on $C_{1}, \cdots, C_{w}$. Actually if $X$ is contained in $\left(^{*}\right)$ with the multiplicity $x$, then

$$
X(\sigma)=x \text { for } \sigma \in C_{i}
$$

and this multiplicity $x$ is characterized by the relations

$$
D g(X) \equiv x(\bmod n) \text { and }|x| \leqq(n-1) / 2 .
$$

In particular $X$ vanishes on $C_{1}, \cdots, C_{w}$ if and only if the degree $D_{g}(X)$ is divisible by $n$.

The relation $|x| \leqq(n-1) / 2$ is a consequence of the equation $\left({ }^{* *}\right)$, since $|x| \leqq x^{2} \leqq l \leqq(n-1) / 2$ (we have assumed $w \geqq 2$ ).

In particular we have

(VI) If $X$ and $Y$ are nonexceptional characters of the same degree then $X$ and $Y$ are contained in $\left(^{*}\right)$ with the same multiplicity.

A similar consideration may be applied to the $A$-characters. Let $\Theta=\Theta_{i}$ be an $A$-character. The character $\theta_{i}$ is a sum of $l$ linear characters of $A: \theta_{i}=\sum^{\prime} \xi^{\prime}$. If a linear character $\xi$ of $A$ is not in $\theta_{i}$, then the induced character $\xi^{*}$ of $G$ is one of $\theta_{1}^{*}, \cdots$, but not $\theta_{i}^{*}$. Let $\alpha$ be the multiplicity of $\Theta$ in $\theta_{j}^{*}(j \neq i)$. Then $\alpha$ is independent of $j$ and the 
multiplicity of $\Theta$ in $\theta_{i}{ }^{*}$ is $\alpha+\epsilon$. Hence if $\beta$ is the multiplicity of $\Theta$ in $\theta_{0}^{*}$, then $\Theta$ is contained in $\theta_{0}^{*}-\theta_{i}{ }^{*}$ with the multiplicity $\beta-\alpha-\epsilon$ and

$$
\Theta / A=\beta \xi_{0}+\alpha \sum \xi+\epsilon \sum^{\prime} \xi^{\prime}
$$

where the third summation extends over $l$ linear characters $\xi^{\prime}$ contained in $\theta_{i}$. Hence if $\sigma \neq 1$ is an element of $A$,

$$
\Theta_{i}(\sigma)=\beta-\alpha+\epsilon \theta_{i}(\sigma) .
$$

We have now the following proposition.

$$
\sum_{\sigma \neq 1, \sigma \in A}\left|\Theta_{i}(\sigma)\right|^{2} \geqq l(n-l) .
$$

From the above consideration we get $\Theta_{i}(\sigma)=\epsilon \theta_{i}(\sigma)+a$ for $\sigma \in A$, $\sigma \neq 1$, where $a$ is a rational integer. Hence

$$
\begin{aligned}
\sum_{\sigma \neq 1, \sigma \in A}\left|\Theta_{i}(\sigma)\right|^{2} & =\sum_{\sigma \neq 1, \sigma \in A}\left(\epsilon \theta_{i}(\sigma)+a\right)\left(\epsilon \theta_{i}\left(\sigma^{-1}\right)+a\right) \\
& =\sum_{\sigma}\left|\theta_{i}(\sigma)\right|^{2}+\epsilon a \sum_{\sigma}\left(\theta_{i}(\sigma)+\theta_{i}\left(\sigma^{-1}\right)\right)+a^{2}(n-1) \\
& =n l-l^{2}-2 \epsilon a l+a^{2}(n-1) \geqq l(n-l),
\end{aligned}
$$

since $a^{2} w-2 \epsilon a \geqq 0$ for $w \geqq 2$ and any integer $a$.

3. Let $G$ be a nonabelian simple group satisfying the condition (W) of the first section. Then each maximal abelian subgroup of $G$ is a subgroup of the type discussed in the $\S 2$. If $A$ and $B$ are two maximal abelian subgroups of $G$, and if $A \cap B \neq e$, then we can take an element $\sigma \neq 1$ of $A \cap B$. Then both $A$ and $B$ are contained in the centralizer of $\sigma$ which has been assumed to be abelian. Hence by maximality of $A$ and $B$ we conclude $A=B$. Thus two distinct maximal abelian subgroups have only the identity in common: in other words maximal abelian subgroups make up a partition of $G$.

The totality of maximal abelian subgroups are divided into classes of conjugate subgroups. Select one representative from each conjugate class and let $A_{1}, A_{2}, \cdots, A_{8}$ be the complete system of such representatives. We shall denote by $N_{i}$ the normalizer of $A_{i}$ in $G$ and let

$$
\left[A_{i}: e\right]=n_{i} \text { and }\left[N_{i}: A_{i}\right]=l_{i} \quad(i=1,2, \cdots, s) .
$$

Each $A_{i}$ is the centralizer of any element $\neq 1$ of $A_{i}$, and hence

$$
w_{i}=\left(n_{i}-1\right) / l_{i}
$$

is an integer.

Our object is to show that the order of $G$ is even. By way of con- 
tradiction we shall assume that the order $g$ of $G$ is odd. The essential consequence from this hypothesis is the following:

For each $i, w_{i}$ is an even integer; in particular $w_{i} \geqq 2$.

We may apply the argument and results in the preceding section for each $A_{i}(i=1,2, \cdots, s)$. We have therefore exactly $w_{i}$ exceptional characters associated with $A_{i}$ for each $i$. By (III) of the $\$ 2$, exceptional characters assciciated with $A_{i}$ are not exceptional for $A_{j}(j \neq i)$. Hence we have $\sum_{i=1}^{s} w_{i}$ characters of $G$ which are exceptional for some of $A_{1}, \cdots, A_{s}$. On the other hand, every element is conjugate to some element of $A_{1}, \cdots, A_{s}$ and each $A_{i}$ contributes exactly $w_{i}$ conjugate classe:s of $G$. Hence $G$ has exactly $1+\sum_{i=1}^{s} w_{i}$ conjugate classes. By a main theorem of group characters, the total number of distinct irreducible characters of a finite group $G$ is equal to the number of conjugate classes in $G$. Hence we have obtained all the irreducible character of $G$ except the principal one by taking exceptional characters for $A_{1}, \cdots, A_{s}$. This means that every nonprincipal characters of $G$ is exceptional for some $A_{i}$.

Assume that the notation is so chosen that $n_{\mathrm{s}}$ is the smallest integer among the $n_{i}$ 's and in order to simplify the formula we write

$$
n_{s}=n, \quad l_{s}=l \quad \text { and } w_{s}=w .
$$

Moreover we assume, in suitably chosen notations, that the degree $d$ of the $A_{\boldsymbol{e}}$-characters is divis:ble by $n_{i}$ with $i \leqq t$ but not divisible by $n_{j}$ $(s>t)$. The relation $\left(^{*}\right)$ of the preceding section is now written as

$$
\Gamma=1-\epsilon\left(\Theta_{i}+a \sum_{k=1}^{w} \Theta_{k}+\sum_{\mu} x_{\mu} X_{\mu}\right.
$$

where the $\Theta_{k}$ 's are $A_{s}$-characters and the $X_{\mu}$ 's are not. By (I) of the $\$ 2 A_{i}$-characters $(i<s)$ have the same degree and hence by (VI) they are contained in $\Gamma$ with the same multiplicity. Let $\left\{\Theta_{j}^{i}\right\}$ be the totality of $A_{i}$-characters $\left(i=1, \cdots, s-1 ; j=1, \cdots, w_{i}\right)$. Then we can write $\Gamma$ as

$$
\Gamma=1-\epsilon \Theta_{i}+a \sum_{k=1}^{w} \Theta_{k}+\sum_{\mu=1}^{s-1} x_{\mu} \sum_{j=1}^{w_{\mu}} \Theta_{j}^{\mu}
$$

Hence $\Gamma$ will vanish on elements of $A_{1}, \cdots, A_{s-1}$. Suppose $x_{i}=0$ for some $i \leqq t$. Then $\Gamma$ does not contain $A_{i}$-characters. Since $i \leqq t$, it follows from $(\mathrm{V})$, that $\Theta_{k}(\sigma)=0$ and each $\Theta_{j}^{\mu}(\sigma)$ is an integer $y_{\mu}$ for $\sigma \neq 1$ of $A_{i}$. Hence $\Gamma(\sigma)=0$ is now read as

$$
0=1+\sum_{\mu \neq i} x_{\mu} y_{\mu} w_{\mu}
$$


which is impossible, since $x_{\mu}, y_{\mu}, w_{\mu}$ are integers and all the $w_{\mu}$ 's are even. Hence we conclude that $x_{i} \neq 0$ for $i=1, \cdots, t$. The relation $\left({ }^{*}\right)$ of the preceding section shows

$$
\begin{aligned}
l & =a^{2}(w-1)+(a-\epsilon)^{2}+\sum_{\mu} x_{\mu}^{2} w_{\mu} \\
& \geqq 1+\sum_{i=1}^{t} x_{i}^{2} w_{i} .
\end{aligned}
$$

Since each $x_{i}(i=1, \cdots, t)$ is a nonvanishing rational integer we conclude

$$
l-1 \geqq \sum_{i=1}^{t} w_{i}
$$

The orthogonality relation for $\Theta=\Theta_{i}$ may be written as

$$
\begin{aligned}
g & =\sum_{\sigma \in G}|\Theta(\sigma)|^{2} \\
& =d^{2}+\sum_{i=1}^{s}\left(g / n_{i} l_{i}\right) \sum_{\sigma \neq 1, o \in A_{i}}|\Theta(\sigma)|^{2} .
\end{aligned}
$$

If $i \leqq t$, we have by (V) $\Theta(\sigma)=0$ for $\sigma \neq 1$ of $A_{i}$. Thus

$$
\sum_{\sigma \neq 1, \sigma \in A_{i}}|\Theta(\sigma)|^{2}=0 \quad \text { for } i=1,2, \cdots, t .
$$

If $t<i<s$, then $\Theta(\sigma)$ is a nonvanishing rational integer by (V). Hence

$$
\sum_{\sigma \neq 1, \sigma \in A_{i}}|\Theta(\sigma)|^{2} \geqq n_{i}-1 \quad \text { for } i=t+1, \cdots, s-1 .
$$

For $i=s$, we have by (VII)

$$
\sum_{\sigma \neq 1, \sigma \in A_{i}}|\Theta(\sigma)|^{2} \geqq l(n-l) .
$$

Hence we conclude that

$$
g \geqq d^{2}+\sum_{i=t+1}^{s-1}\left(g / n_{i} l_{i}\right)\left(n_{i}-1\right)+(g / n)(n-l) .
$$

On the other hand, every element of $G$ is conjugate to some element of $A_{1}, \cdots, A_{\mathrm{s}}$. Hence

$$
g=1+\sum_{i=1}^{s}\left(g / n_{\imath} l_{i}\right)\left(n_{i}-1\right) .
$$

Hence 


$$
1+\sum_{i=1}^{t}\left(g / n_{i} l_{i}\right)\left(n_{i}-1\right)+(g / n l)(n-1) \geqq d^{2}+(g / n)(n-l) .
$$

It follows now

$$
\sum_{i=1}^{t}\left(n_{i}-1\right) / n_{i} l_{i}+\left(n-1+l^{2}\right) / n l \geqq 1+\left(d^{2}-1\right) / g .
$$

By definition $\left(n_{i}-1\right) / l_{i}=w_{i}, n_{i} \geqq n$ for $i=1,2, \cdots, t$. Hence

$$
\left(\sum_{i=1}^{t} w_{i}\right) / n \geqq \sum_{i=1}^{t}\left(n_{i}-1\right) / n_{i} l_{i} \text {. }
$$

We have already obtained the inequality

$$
l-1 \geqq \sum_{i=1}^{t} w_{i}
$$

Using these estimations we get

$$
(l-1) / n+\left(n-1+l^{2}\right) / n l \geqq 1+\left(d^{2}-1\right) / g .
$$

Since we have assumed that $G$ is a nonabelian simple group, $d$ is greater than one as being the degree of a nonprincipal character. Hence

$$
(l-1) / n+\left(n-1+l^{2}\right) / n l>1
$$

Since $n-1=w l$, we may write this strict inequality in the form

$$
\begin{gathered}
l-1+w+l>w l+1, \quad 2(l-1)+w(1-l)>0 \\
(l-1)(2-w)>0 .
\end{gathered}
$$

This is however impossible, because $l \geqq 1$ and $w \geqq 2$.

Thus we have shown the validity of the following theorem.

THEOREM. If a nonsolvable group $G$ satisfies the condition (W) of the first section, then the order of $G$ must be even.

Together with the result in [2], we may conclude the following:

THEOREM. The nonsolvable group $G$ satisfying the condition $(\mathrm{W})$ is one of the linear groups $\operatorname{LF}\left(2,2^{\mu}\right)$.

4. In this section we shall consider a nonabelian simple group $G$ such that every maximal subgroup of $G$ contains only nilpotent proper subgroups. Our purpose is to show that such a group is isomorphic with the alternating group on 5 letters.

First of all, we shall show that the Sylow subgroups of $G$ are independent, i.e. their intersection is $\{e\}$. By way of contradiction, we 
shall assume the maximal $p$-intersection $D$ is not the unit subgroup. The normalizer $N(D)$ is not nilpotent. Hence $N(D)$ is a maximal subgroup, which is not nilpotent. By assumption, every proper subgroup of $N(D)$ is nilpotent. Hence by a theorem of Iwasawa [5], a $p$-Sylow subgroup $T_{p}$ of $N(D)$ is cyclic. Since $N(D)$ is maximal, the normalizer of $T_{p}$ is contained in $N(D)$. It follows now that $T_{p}$ is a $p$-Sylow subgroup of $G$, and is contained in the center of its normalizer. By a theorem of Burnside ( $[3$, p. 237] $) G$ is not simple against our assumption. Hence the Sylow subgroups of $G$ are independent.

Next we shall show that any maximal subgroup of $G$ is not nilpotent. If a maximal subgroup $M$ is nilpotent, then $M$ is the normalizer of the center of Sylow subgroup of $M$. We have already shown that Sylow subgroups are independent. Hence for all prime divisors of the order of $G, G$ is $p$-normal in the sense of Grün (cf. [4]). By a theorem of Grün we have an isomorphism between $p$-commutator factor groups $G / G^{\prime}(p)$ and $N / N^{\prime}(p)$, where $N$ is the normalizer of the center of a $p$-Sylow subgroup. We may therefore take $M=N$. Then $M / M^{\prime}(p) \neq e$ for some $p$ and this implies that the commutator subgroup of $G$ is a proper subgroup, which is not the case. Hence $M$ is not nilpotent.

Let $M$ be any maximal subgroup of $G$. Then $M$ is not nilpotent, but all proper subgroups of $M$ are nilpotent. Since Sylow subgroups of $G$ are independent, the order of $M$ has the form $p^{\alpha} q$ where $p$ and $q$ are distinct prime numbers. This follows from the result of Iwasawa [5]. Furthermore the $p$-Sylow subgroup $S_{p}$ of $M$ is a normal subgroup of $M$. If $T_{p}$ is the commutator subgroup of $S_{p}$, then $T_{p}$ and a $q$-Sylow subgroup $T_{q}$ of $M$ generate a nilpotent subgroup $U$. We want to show $T_{p}=e$. By way of contradiction, assume $T_{p} \neq e$. Let $S_{q}$ be a $q$-Sylow subgroup of $G$ containing $T_{q}$. If $\sigma \in T_{p}, \sigma S_{q} \sigma^{-1} \supseteq \sigma T_{q} \sigma^{-1}$. $=T_{q}$. Since the Sylow subgroups of $G$ are independent, $\sigma S_{q} \sigma^{-1}=S_{q}$. Let $N$ be a maximal subgroup of $G$ containing the normalizer of $S_{q}$. Then $N \supseteq S_{q} \cup T_{p}$. If $N=M$, then $S_{q}=T_{q}$ and $S_{q}$ is contained in the center of the normalizer of $S_{q}$. This is impossible by a theorem of Burnside (loc. cit.). Hence $N \neq M . N$ is again not nilpotent but every proper subgroup of $N$ is nilpotent. Hence by Iwasawa's theorem (loc. cit.) $N=S_{q} \cup 1_{p}$ and $\left[T_{p}: e\right]=p$; in particular, $S_{q} \neq T_{q} . U=T_{p} \cup T_{q}$ is therefore a cyclic group of order $p q$ and $U=M \cap N$. The normalizer of $U$ is contained in the normalizer of $T_{p}$ which is $M$ and at the same time is in the normalizer of $T_{q}$ which is $N$. Hence the normalizer of $U$ coincides with $U=M \cap N$. Denote by $p^{\alpha}, q^{\beta}$ the orders of $S_{p}$ and $S_{q}$ respectively. Then $G$ contains $\left(g / p^{\alpha} q\right)\left(p^{\alpha}-1\right)$ elements with orders a power of $p$, where $g=[G: e]$. Similarly conjugate subgroups of $S_{q}$ contain 
$\left(g / q^{\beta} p\right)\left(q^{\beta}-1\right)$ elements other than the identity. Since conjugate subgroups of $U$ contain $(g / p q)(p q-p-q+1)$ elements of order $p q$, we have an inequality:

$1+\left(g / p^{\alpha} q\right)\left(p^{\alpha}-1\right)+\left(g / q^{\beta} p\right)\left(q^{\beta}-1\right)+(g / p q)(p q-p-q+1) \leqq g$,

or

$$
(1 / g)+(1 / p q) \leqq(1 / p q)\left(\left(1 / p^{\alpha-1}\right)+\left(1 / q^{\beta-1}\right)\right) .
$$

Since $\alpha \geqq 2, \beta \geqq 2$, we get

$$
1<(1 / p)+(1 / q) .
$$

This inequality is however impossible. Hence $T_{p}$ must be the unit subgroup.

This shows that every maximal subgroup of $G$ contains only abelian proper subgroups. It is now easy to verify that the condition (W) of the opening section is satisfied. By our main theorem the order of $G$ must be even. Hence by a theorem of Redéi [6], $G$ is isomorphic with the alternating group on five letters.

TheOREM. Let $G$ be a nonabelian simple group. If every maximal subgroup of $G$ contains only nilpotent proper subgroups, then $G$ is isomorphic with the alternating group on five letters.

\section{REFERENCES}

1. R. Brauer and K. A. Fowler, On groups of even order, Ann. of Math. vol. 62 (1955) pp. 565-583.

2. R. Brauer, M. Suzuki and G. E. Wall, (to appear).

3. W. Burnside, Theory of groups, 1911.

4. O. Grün, Beiträge zur Gruppentheorie I, J. Reine Angew. Math. vol. 174 (1936) pp. 1-14.

5. K. Iwasawa, Über die Structur der endlichen Gruppen, deren echte Untergruppen sämtlich nilpotent sind, Proceedings of the Physics-Mathematical Society of Japan vol. 23 (1941) pp. 1-4.

6. L. Redéi, Ein Satz über die endlichen einfachen Gruppen, Acta. Math. vol. 84 (1950) pp. 129-153.

7. M. Suzuki, On finite groups with cyclic Sylow subgroups for all odd primes, Amer. J. Math. vol. 77 (1955) pp. 657-691.

8. L. Weisner, Groups in which the normaliser of every element except the identity is abelian, Bull. Amer. Math. Soc. vol. 31 (1925) pp. 413-416.

UNIVERSITY OF ILLINOIS 